\title{
Method for the solution of the nucleation problem in arbitrary mesoscopic superconductors: Theory and application
}

\author{
Paulo J. Pereira, ${ }^{1}$ Victor V. Moshchalkov, ${ }^{1}$ and Liviu F. Chibotaru ${ }^{2}$ \\ ${ }^{1}$ INPAC-Institute for Nanoscale Physics and Chemistry, Katholieke Universiteit Leuven, Celestijnenlaan 200 D, B-3001 Leuven, Belgium \\ ${ }^{2}$ Division of Quantum and Physical Chemistry and INPAC, Institute for Nanoscale Physics and Chemistry, Katholieke Universiteit Leuven, \\ Celestijnenlaan 200F, B-3001 Leuven, Belgium
}

(Received 11 April 2012; published 21 November 2012)

\begin{abstract}
We present a method for finding the condensate distribution at the nucleation of superconductivity for arbitrary polygons. The method is based on conformal mapping of the analytical solution of the linearized Ginzburg-Landau problem for the disk and uses the superconducting gauge for the magnetic potential proposed earlier. As a demonstration of the method's accuracy, we calculate the distribution of the order parameter in regular polygons and compare the obtained solutions with available numerical results. As an example of an irregular polygon, we consider a deformed hexagon and prove that its calculation with the proposed method requires the same level of computational efforts as the regular ones. Finally, we extend the method over samples with arbitrary smooth boundaries. With this, we have made simulations for an experimental sample. They have shown perfect agreement with experimental data.
\end{abstract}

DOI: 10.1103/PhysRevE.86.056709

PACS number(s): 02.70.-c, 74.20.De, 11.15.Bt

\section{INTRODUCTION}

Recent capabilities of creating nano-sized samples provide the possibility to study vortex matter at the nanoscale. The coherent length of order parameters for the known superconductor materials is typically of this order of magnitude. At this scale, theory predicted new superconducting vortex states, like the giant vortex [1] and antivortex [2], created by the confinement of the condensate. This makes the ability to simulate the superconducting order parameter in samples of general shapes important, as is usually the case with experimental samples.

The linearized Ginzburg-Landau (LGL) equation describes the distribution of the superconducting order parameter $(\Psi)$ close to the normal-superconducting phase boundaries, $T_{c}\left(H_{c 2}\right)$, i.e., at the nucleation point, as function of temperature and magnetic field $[3,4]$. Besides, it was shown that the solutions of this equation can be used to solve the nonlinear Ginzburg-Landau (GL) problem [5,6].

The LGL problem is defined by the equations

$$
\begin{gathered}
\frac{1}{2 m}(-i \hbar \vec{\nabla}-2 e \vec{A})^{2} \Psi=\alpha \Psi, \\
\left.(-i \hbar \vec{\nabla}-2 e \vec{A})\right|_{\text {n.b. }} \Psi=0,
\end{gathered}
$$

where $\vec{A}$ is the vector potential corresponding to the applied magnetic field $(\vec{H}=\nabla \times \vec{A}), \Psi$ is the superconducting order parameter, $\alpha$ is the condensation energy density (which in the linearized Ginzburg-Landau is an eigenvalue to be determined), and n.b. means normal to the boundary. Equation (1) looks like a Schrödinger equation for a particle in the magnetic field. Equation (2) is the boundary condition for superconductivity which differs from the one of particle in the box problem (or quantum billiard) for which $\Psi=0$ at the boundary. The boundary condition (2) plays an important role for mesoscopic superconductors, like loops [7], disks [1], triangles [8], squares [2], and rectangles [9].

The most common method to solve the GL problem in superconductivity (the linearized GL equation, nonlinear GL equation, the GL equation coupled with the magnetic field equation, the time-dependent GL, and the two-component GL) is the finite difference method [10-14]. However, comparison with analytical and semianalytical methods applied to the LGL equation have shown that the finite difference method needs a great number of points (with grids of at least 201 by 201 points [15]) to become sufficiently accurate to obtain vortex features like an antivortex in a square [2]. Still, analytical and semianalytical methods are only applicable, to the best of our knowledge, to a few bounded geometries like the circle [16-18], square [2,19], rectangle [9], and equilateral triangle [8] and annular [20] geometries.

Most of the newly accessible bounded geometries by semianalytical methods were solved by a superconducting gauge method [21,22]. In this method, the boundary condition of the LGL problem, Eq. (1), is simplified to the Neumann boundary condition, $\left.\vec{\nabla}\right|_{\text {n.b. }} \Psi=0$, by fixing the gauge of the vector potential, $\left.\vec{A}\right|_{\text {n.b. }}=0$. This is called the superconducting gauge [21]. In the superconducting gauge approach, the gauge function, $S(\vec{r})$, is found from the condition

$$
\begin{gathered}
\left.\vec{\nabla}\right|_{\text {n.b. }} S=-\left.\overrightarrow{A_{0}}\right|_{\text {n.b. }}, \\
\vec{A}=\overrightarrow{A_{0}}+\vec{\nabla} S,
\end{gathered}
$$

where $\vec{A}_{0}=\frac{1}{2} \vec{H} \times \vec{r}$ and $\vec{H}$ is a homogeneous magnetic field applied normally to the surface of polygons. To find the gauge function $S$, an analytical method was developed in previous publications [21,22]. An ansatz for $S$, expressed in the polar coordinates, was used,

$$
S(r, \theta)=R(r) \sin (N \theta),
$$

where $N$ is the number of vertices in the polygon. With this ansatz, Eq. (3) becomes a first-order differential equation in $R(\theta)$. By solving it and analytically expanding the solution, $R(r)$ is obtained. This method provides for the triangle and square simple explicit analytical expressions for the vector potential. A general expression was deduced for arbitrary regular polygons. After simplifying the boundary condition, 
Eqs. (3) and (4), the eigenfunctions of the Laplacian operator (a particle in the box problem) were used as basis to solve the LGL equation (1). The solutions of the LGL equation can afterwards form a basis to solve the full nonlinear GinzburgLandau problem. The advantage of this approach over the finite element method is the use of a few low-lying eigenfunctions of the Laplacian operator in the LGL problem and only a few of the low-lying LGL solutions to represent the solution of the full GL problem.

Recently, a numeric method [23] was developed to solve the LGL problem for bounded geometries using the finite element method and the superconducting gauge approach. The solutions for this problem were found for different geometries, including some highly symmetric geometries, like the square, triangle, pentagon, and five-pointed star. This approach is more efficient than the finite difference method; however, it lacks the compactness and spacial resolution of the semianalytical methods.

As already mentioned, the quantum billiard problem is equivalent to the LGL problem in a zero magnetic field with Dirichlet boundary conditions. This problem was solved by various methods [24-28]. Some of these methods [26] showed that conformal mapping is an important tool to find eigenfunctions of the Laplacian operator with Dirichlet boundary conditions. The main reason is that this transformation leaves invariant the form of the Laplace equation, i.e., the mapped solution of the Laplace equation in a given geometry is the a solution of the Laplace equation in the mapped space. Another important feature is that these transformations preserve the angles, which means that a function compliant with the Neumann boundary condition is mapped into a new function compliant with the same boundary conditions, in the new geometry. This makes the conformal mapping a perfect tool to solve the LGL problem with Neumann boundary conditions.

In this paper, we present a new method to solve the LGL equation for general polygons based on conformal mapping. Equation (3) defined in the polygon was mapped into the circle and solved there analytically. The solutions for the disk of the LGL with an out magnetic field then were mapped into the polygon and used as a basis set to solve the LGL equation. The solutions for some regular polygons were calculated in order to compare with the previous numerical solutions and assess the accuracy. These solutions share a very close correspondence between them. Some of the regular polygons have already known solutions $[2,8,23]$ for the LGL equation. These were also applied to better test the quality of the algorithm.

The conformal map used in our method is the a variant of the Schwartz-Christoffel (SC) transformation [29]. A straightforward modification of the original mapping makes this go from the interior of a circle into a general polygon. The modified SC transformation from a circle is obtained by the following function:

$$
\begin{gathered}
w(z)=C \int \prod_{i=0}^{N}\left(1-\frac{z}{z_{i}}\right)^{\alpha_{i}-1} d z+B, \\
w=x+i y, \\
z=u+i v,
\end{gathered}
$$

where $C$ and $B$ are complex constants (related with the size and position of the polygon, respectively), $z_{i}$ is the point in the circumference that is mapped into the vertex $i$ of the polygon, $\alpha_{i}$ is the angle of the polygon's vertex $i, x$ and $y$ are Cartesian coordinates of the problem space (polygon), and $u$ and $v$ are Cartesian coordinates of the auxiliary space (circle). This expression can be simplified in the case of regular polygons. We have used the Schwartz-Christoffel toolbox [30] (an open-source MATLAB package) to compute the SC mapping.

\section{DESCRIPTION OF THE METHOD}

We propose to solve the LGL problem in a generic polygon with the eigenfunctions of the Laplacian operator (with Neumann boundary condition), defined on the circle, as basis functions. We also propose to fix the gauge of the vector potential, which reduces the boundary condition of the Ginzburg-Landau problem to the Neumann boundary condition, by solving Laplace equation with a general Neumann boundary condition (which can be found by the Dini integral formula [31]).

In the development of this method, we started by calculating the eigenfunctions of the Laplacian operator in the circle (with Neumann boundary condition). These well-known solutions are

$$
\Psi_{(l, n)}(r, \theta)=J_{l}\left(u_{(l, n)}^{\prime} r\right) e^{i l \theta}, \quad J_{l}^{\prime}\left(u_{(l, n)}^{\prime}\right)=0,
$$

where $u_{(l, n)}^{\prime}$ is the $n$ zero of the $l$ Bessel function derivative and $J_{l}\left(u_{(l, n)}^{\prime} r\right)$ is the $l$ Bessel function rescaled to match the Neumann boundary conditions. The solutions for the circle (auxiliary space) are mapped to the general polygon (original space) by the SC mapping Eq. (6). We have represented the functions with a mesh in the original space. Afterwards, this set of functions was orthogonalized via the Löwding orthogonalization process [32]. The new functions then were used as a basis for the LGL problem. To apply the functions as a basis for the LGL equation, the superconducting gauge needs, first, to be fixed using the condition (3).

In the future we are also planning to expand the method to the second Ginzburg-Landau equation. The Coulomb gauge is the typical gauge for the second Ginzburg-Landau equation. If we want to satisfy (3) while keeping the Coulomb gauge, the function $S$ must be a solution of the problem

$$
\begin{gathered}
\Delta S=0 \\
\left.\vec{\nabla}\right|_{\text {n.b. }} S=-\left.\vec{A}\right|_{\text {n.b. }},
\end{gathered}
$$

where $\vec{A}$ is a general vector potential. In general, this problem is only solvable when

$$
\left.\int_{\text {boundary }} \vec{A}\right|_{\text {n.b. }}=0,
$$

where the integration is made over the boundary. This condition is fulfilled if the original vector potential is in the Coulomb gauge, $\vec{\nabla} \cdot \vec{A}=0$, i.e., the superconducting gauge condition, $\left.\vec{A}\right|_{\text {n.b. }}=0$, is complemented by the Coulomb gauge. To solve the new problem, we have mapped the equation back 

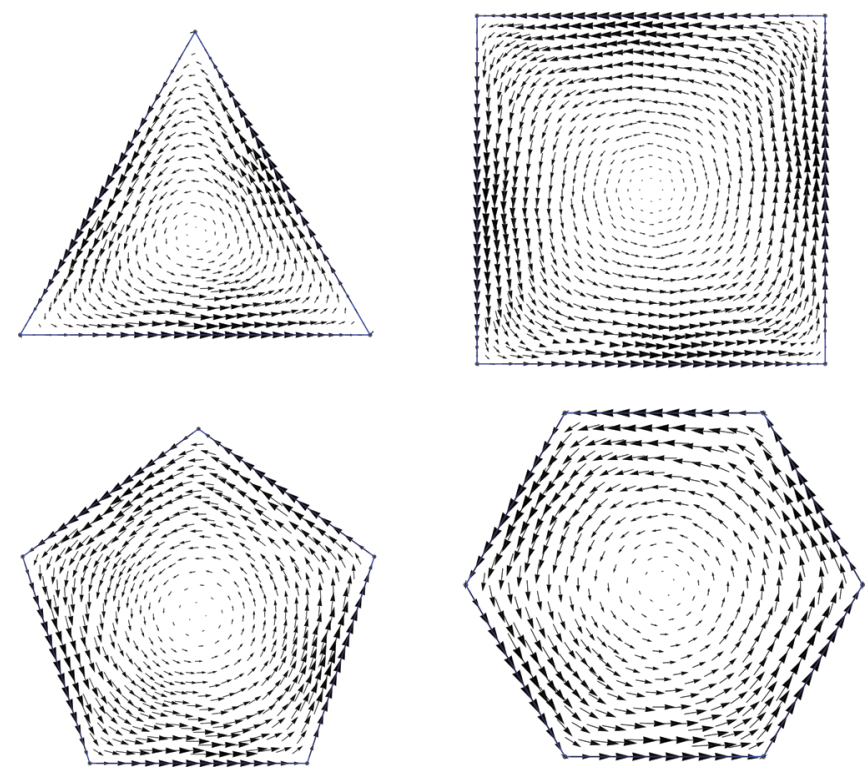

FIG. 1. (Color online) Plots of the vector potential with the superconducting gauge fixed by the method described in the text.

to the auxiliary space (circle), making it

$$
\begin{gathered}
\Delta S(u, v)=0, \\
\left.\vec{\nabla} S(u, v)\right|_{\text {n.b. }}=-\left.\left|w^{\prime}(z)\right| \vec{A}(u, v)\right|_{\text {n.b. }},
\end{gathered}
$$

where

$$
\left|w^{\prime}(z)\right|=\prod_{i=0}^{N}\left|\left(1-\frac{z}{z_{i}}\right)^{\alpha_{i}-1}\right| .
$$

This can be done because the Laplace equation is invariant under conformal mappings. For the circle there is a general analytical solution for the Laplace problem with generic Neumann boundary condition. That solution is given by the Dini integral formula [31]

$$
\begin{gathered}
S(u(r, \theta), v(r, \theta))=\frac{R}{4 \pi} \int_{\phi=0}^{2 \pi}-\left.\left|w^{\prime}(z)\right| \vec{A}(u, v)\right|_{\text {n.b. }} \\
\log \left(r^{2}-2 r R \cos (\theta-\phi)+R^{2}\right) / R^{2} d \phi,
\end{gathered}
$$

for the unitary circle, i.e., $R=1$, where $\phi$ is an integration angle. This method makes it possible to fix the superconducting gauge for any magnetic field distribution, such that the vector potential is tangential to the boundary. Figure 1 shows the vector potential with the fixed gauge for the triangle, square, pentagon, and hexagon and Fig. 6 below presents the vector potential with the fixed gauge for a deformed polygon.

\section{SOLUTIONS FOR REGULAR POLYGONS}

As a test for our approach, we have calculated the lower state of the LGL equation for the regular polygons: triangle, square, pentagon, and hexagon. As a basis set, 45 functions were used for each irreducible representation of the polygons [22], taken from Eq. (9). The solutions for the triangle and square geometries can be compared to the previous publications.

The analytical solution for the circle geometry was also calculated to be compared with the regular polygon solutions.
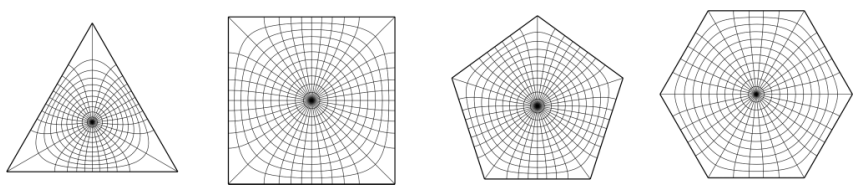

FIG. 2. (Color online) Plots of the curvilinear coordinates lines of the Schwartz-Christoffel mapping. The deformed circular lines are the result of the mapping of equally space circular lines defined in a circle geometry onto to polygons. The radial lines are the result of the mapping of equally spaced radial lines, defined in the circle, onto the polygons.

This analytical solution is given by [17]

$$
\begin{aligned}
\Psi(r, \theta)= & e^{ \pm i L \theta} R^{L / 2} N^{L / 2} \exp \left(\frac{-N R}{2}\right) \\
& \times M\left[-n(L, N), L+1, N R^{2}\right],
\end{aligned}
$$

where $n$ defines the nucleation boundary transition and must satisfy the following equation:

$$
\begin{aligned}
& (L-N) M(-n, L+1, N) \\
& \quad-\frac{2 n N}{L+1} M(-n+1, L+2, N)=0,
\end{aligned}
$$

where $N=\Phi / \Phi_{0}, \Phi$ is the magnetic flux, $\Phi_{0}$ is the magnetic flux quantum, $R=r / r_{0}, r_{0}$ is the radius of the circle, $M$ is the first Kummer function, and $L$ is the vorticity of the state. Figure 2 shows a graphical representation of the mapping for the different geometries. In this representation, some polar coordinate lines of the circle (i.e., lines with fixed radius and lines with fixed angle), regularly spaced, are mapped into the polygons.

Figure 3 shows the $T-H$ nucleation curves for the regular polygons and disk. As usual, cusps are observed in all curves. As the number of vertices in the polygon becomes higher, the critical temperature becomes lower for a fixed magnetic field. Each phase boundary domain between two cusps corresponds to a definite vorticity. Figure 4 shows the vortex patterns for the states of vorticity 1 to 6 for the different geometries. It can be seen that the results for the triangle, square, and pentagon are in agreement with the already known results [2,8,23], indicating that the present method is a natural extension of the previously reported superconducting gauge method [22]. From Fig. 4 we can see the simulation results as the number of vertices $\left(N_{v}\right)$

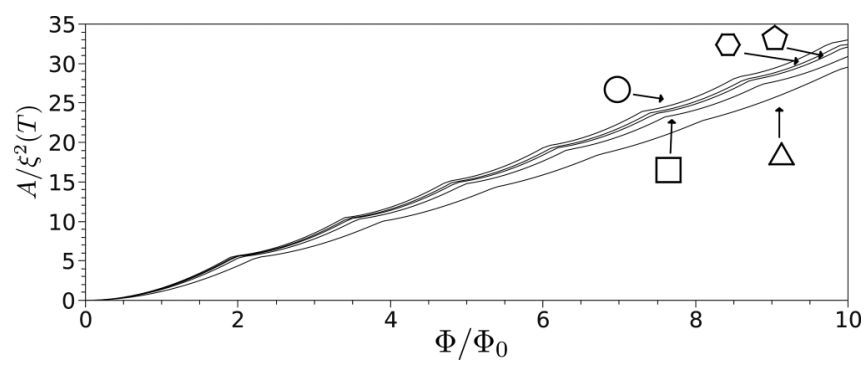

FIG. 3. Plot of the nucleation curve in the $T-H$ space for different geometries: triangle, square, pentagon, hexagon, and circle. $A, \xi(T)$, $\Phi$, and $\Phi_{0}$ denote the area of the sample, the coherence length of the superconducting condensate, the magnetic flux passing thought the sample, and the magnetic flux quantum, respectively. 

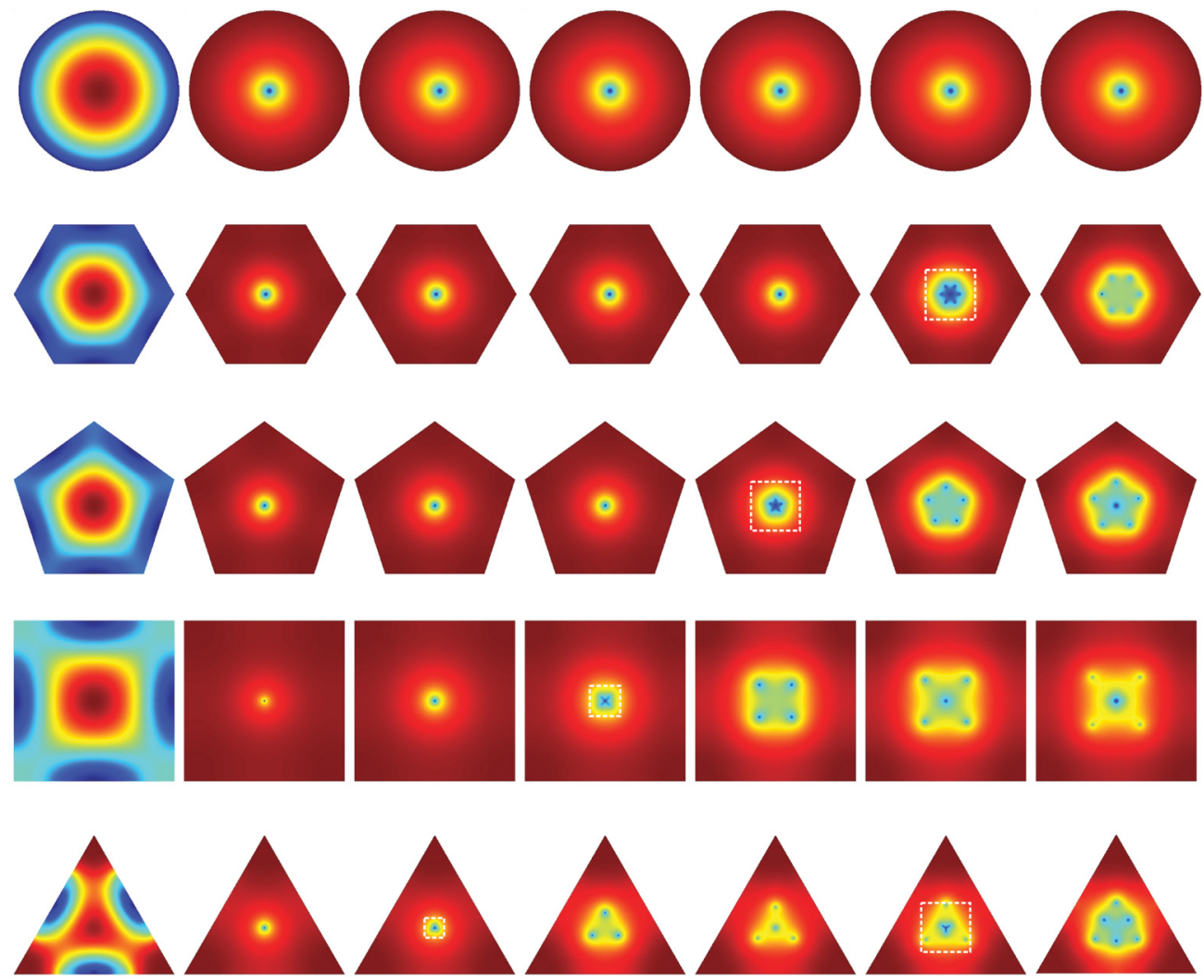

FIG. 4. (Color online) Plots of the distribution of the superconducting order parameter| $\Psi \mid$, in the natural logarithmic scale, for the triangle, square, pentagon, hexagon, and circle geometries for different vorticities $L=0,1,2,3,4$, 5, and 6 (counting from left to right), where the magnetic field in magnetic flux quanta is $\Phi / \Phi_{0}=1,3,4,5.5,7,8,9$, respectively. The color scale goes from red (dark gray) where superconductivity is strong, through yellow (light gray) to blue (dark gray). This color scheme is present in all figures that show the density of the superconducting condensate. The vortices appear as blue (dark gray) spots in the middle of the sample. Figure 5 shows the zooms from the areas of the present figure enclosed by white dashed boxes.

increases (equally spaced) from 1 to 6 and to infinite (circle). We can see that the patterns of the polygons approach the pattern of the circle as the number of vertices increases. This happens quite rapidly with the number of vertices. For the hexagon geometry, almost all the patterns have a central giant vortex just like in the circle, except for the vorticities 5 and 6 (which equals $N_{v}-1$ and $N_{v}$, respectively).

The Meissner states at different geometries also indicate a tendency for the superconducting condensate to become stronger near the corners of the polygon. Thus, one can see from Fig. 4 that, for the triangle geometry in this superconducting state, the superconductivity begins to nucleate strongly around the vertices (it is the place where it nucleates strongly even compared with the middle of the sample). For the square geometry, the condensate is already weaker in the corners than in the middle of the sample.

Figure 4 shows that all considered regular polygons have patterns with one antivortex in the center. These patterns are always associated with the states with a vorticity number equal to the number of vertices minus $1[2,8]$. Since the equation solved is linear, all states must be symmetry compliant. In the states with vorticity equal to the number of vertices $\left(N_{v}\right)$ minus 1 , it seems that having one antivortex in the middle and $N_{v}$ vortices around it is energetically more favorable than having a central giant vortex. Figure 5 shows a zoom of the antivortex patterns, where the antivortex appears in the center of the panels. The state with vorticity $N_{v}$ has always $N_{v}$ vortices dispatched along each radial line that passes through the vertices of the polygon.

\section{IRREGULAR POLYGONS}

As an example of an irregular polygon analyzed by use of the presented method, we decided to solve the problem for a deformed hexagon. First, the superconducting gauge was fixed with the method described above. Figure 6 shows the vector potential, with the gauge adjusted to match the boundary conditions. Afterwards, we calculated the lowest state of the
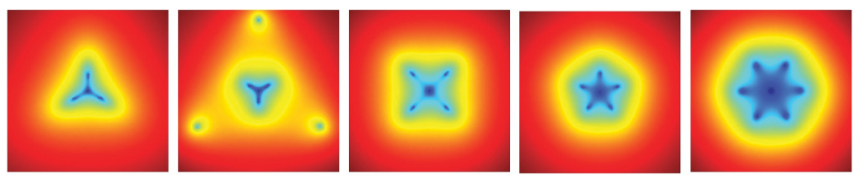

FIG. 5. (Color online) Zoom of the superconductor order parameter patterns with antivortices shown in Fig. 4 by dashed boxes. The color scale differs from the one in the respective panels of the Fig. 4. The patterns correspond, from left to right, to the pairs (geometry, number of vortices, vorticity number): (triangle, 3,2 ); (triangle, 6, 5); (square, 4, 3); (pentagon, 5, 4); (hexagon, 6, 5). 


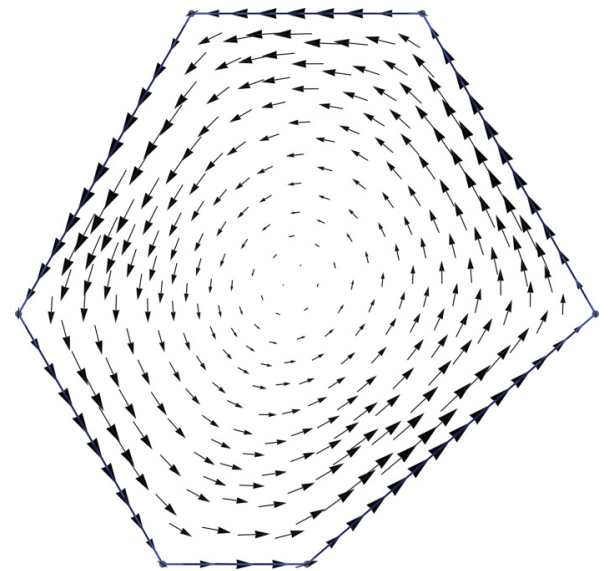

FIG. 6. (Color online) Vector potential with the superconducting gauge fixed so the vector potential lines become parallel to the boundary, as described in the text.

LGL equation for the different vorticities, as shown in Fig. 7. A basis set of 190 functions given by Eq. (9) (for 10 different $n$ and for 19 different $l$ ) was used.

In the Meissner state $L=0$ (Fig. 7), one can observe a stronger nucleation of superconductivity at the boundary. This is strongly correlated with the vertices angles of the polygon. In Fig. 7, one can also see that the vortex positions different markedly from those of the hexagon. There are no giant vortices or antivortices in the patterns, and these tend to be aligned along the line between the left bottom vertex and the top right vertex, as corresponding to the largest length in the sample.

\section{GENERAL SMOOTH BOUNDARY}

To calculate the nucleated condensate density distribution in a general selected shape, for example, of an experimental sample, we can surround this shape by a polygon and then use the vertices of the polygon as control points to match an interior angular coordinate line [i.e., $w\left(R_{s} e^{\theta}\right)$ for a constant $R_{s}<1$ in the auxiliary domain] with the shape.

As an application for general boundaries, we have used the border of an experiment sample displayed as N1 in Fig. 3 of

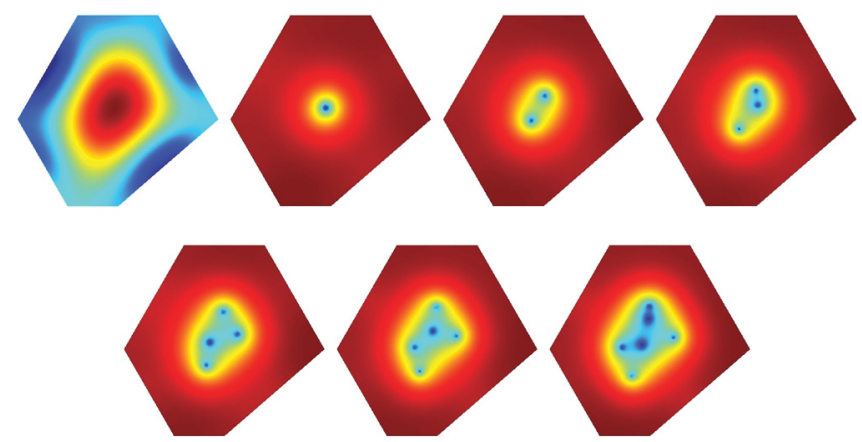

FIG. 7. (Color online) Distribution of the superconducting order parameter, in the natural logarithmic scale, in the deformed hexagon for vorticities $L=0,1,2,3,4,5$, and 6 (counting from left to right, from top to bottom), where the magnetic field in magnetic flux quanta is $\Phi / \Phi_{0}=1,3,4,5.5,7,8,9$, respectively. The vortices appear as blue (dark gray) spots in the middle of the sample.

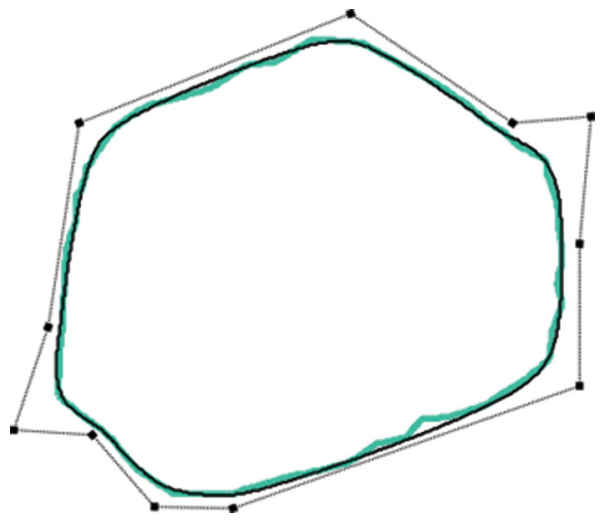

FIG. 8. (Color online) Plots of the experimental sample border defined as N1 in Fig. 1 of Ref. [33], the adjusted curve (created by the method described in the text for the value of $R_{s}=0.95$ ) and the associated polygon, displayed by a thick light green (light gray) line, a full black line (on top of the thick line) and a dotted line (with squares on the corners), respectively.

Ref. [33]. In Fig. 8, the border of experimental sample can be seen as a gray thick line (light green). A smooth curve approximating the boundary of the sample is defined by a circular coordinate line (black solid line), where $R_{s}=0.95$ plotted on top of the experimental border, with the associated polygon (that defines this curve) surrounding it (dotted black line). To define the number of points of the polygon and its location, a small piece of code was developed on top of the Schwartz-Christoffel toolbox [30] software (this already allows us to change, by hand, the number of points and their locations) that draws the angular coordinate line, on top of the experimental border, for a chosen $R_{s}$ in real time.

We have used again the Bessel functions as basis functions, but now they have been rescaled to match the Neumann boundary condition on the corresponding circular coordinate line of the auxiliary domain, i.e., $J_{l}^{\prime}\left(u_{(l, n)}^{\prime} R_{s}\right)=0$. We then orthonormalized this basis by use of the Löwing orthogonalization process, as in the previous sections. The orthonormal basis can be used to express the LGL equation in a matrix form and

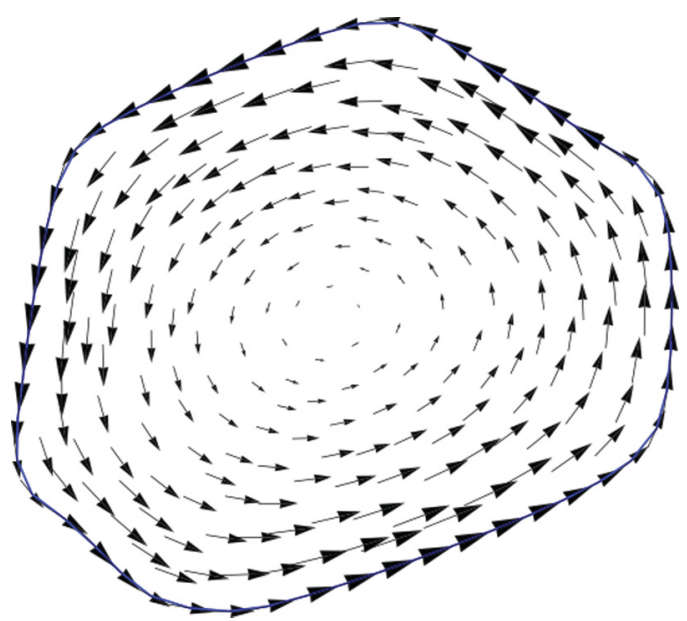

FIG. 9. (Color online) Plot of the vector potential of corresponding to a homogeneous perpendicular magnetic field in the superconducting gauge for the shape defined in Fig. 8. 
its diagonalization gives us the solutions of the equation, but, first, we need to put the vector potential in the superconducting gauge.

To fix the appropriate gauge, i.e., $\left.\vec{A}\right|_{\text {n.b. }}=0$, we must calculate the normal projection (to the boundary) of the magnetic field. We can do this by calculating the normal vector with the conformal map that defines the boundary. The normal vector becomes

$$
\tilde{n}=\frac{w^{\prime}(z) z}{\left|w^{\prime}(z) z\right|}, \quad \vec{n}=(\operatorname{Re}(\tilde{n}), \operatorname{Im}(\tilde{n})),
$$

where $\mathrm{Re}$ and $\mathrm{Im}$ denote the real and imaginary parts, respectively. We note that the normal vector in previous expression is defined in the problem space as function of a point given in the auxiliary space. Then, solving the Dini equation (16) with $R=R_{s}$, one can calculate the function $S$ and then fix the gauge via, i.e., Eq. (3). Figure 9 shows the vector potential in the superconducting gauge for the shape defined in Fig. 8.

\section{APPLICATION TO EXPERIMENTAL EXAMPLES}

In this section, we analyze the simulations made for the shape defined in the previous section and compare them with the experimental data from Ref. [33]. The experimental samples described in that publication are very thin (10 to 8 atomic layers), which puts them in the extreme type II regime. Therefore, the screening currents can be ignored in these samples. This allows for a direct application of our method. The experimental data from Fig. 4 of Ref. [33] are reproduced in the left panel of Fig. 10. Figure 10 (bottom right) displays distributions of the order parameter calculated for different values of the field by use of the method mentioned in the previous section. In the top-right corner of Fig. 10, the location of experimental and theoretical values on the $T-H$ diagram are shown. For these simulations we used 190 basis functions (10 different $n$ times 19 different $l$ ).

We will now contrast the simulations in Fig. 10 with the experimental data in the same figure. The condensate distributions (scanning tunneling microscopy measurements at zero-bias conductance) in the sample (the top-left sample

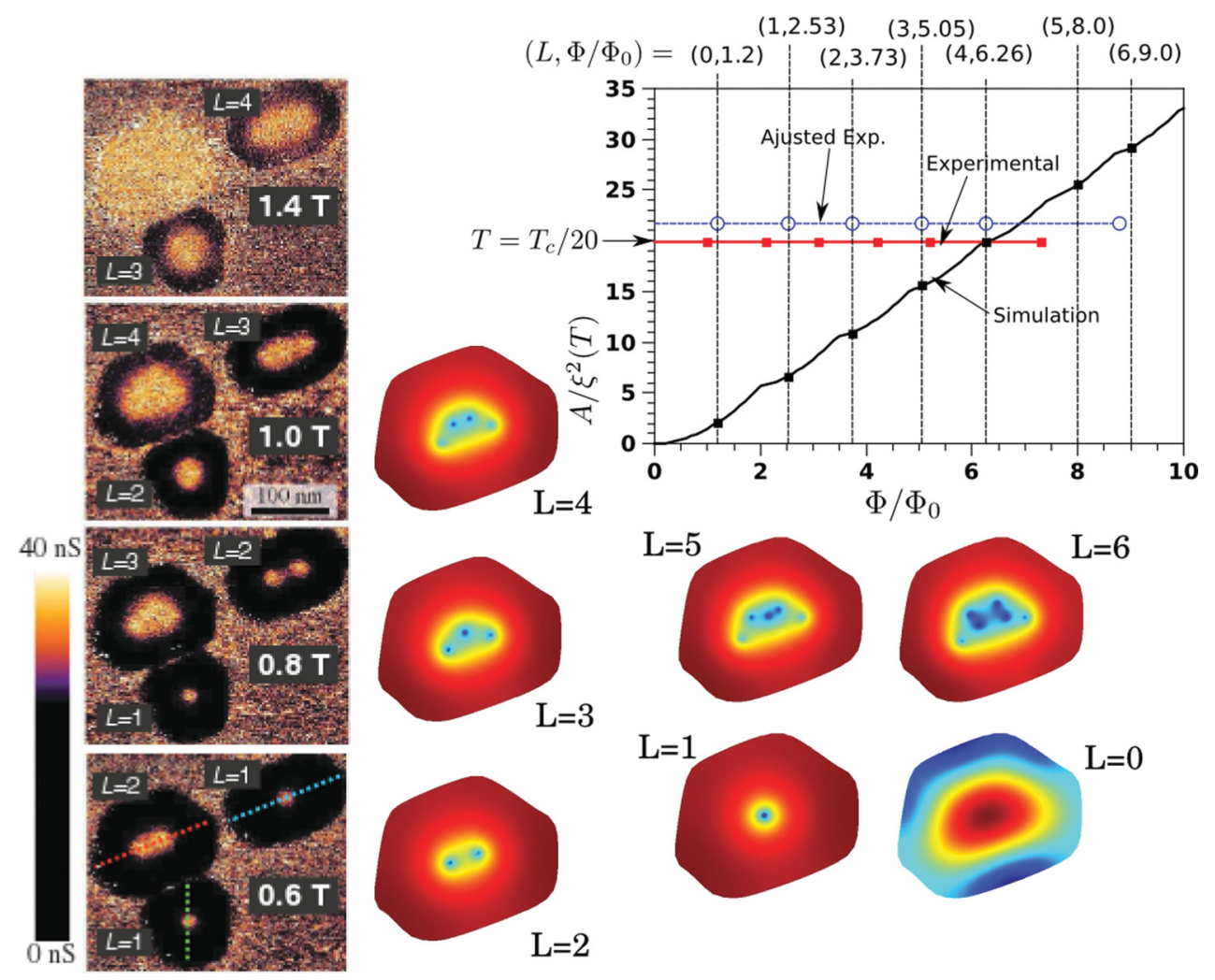

FIG. 10. (Color online) Experimental distributions and simulations to the experimental sample referred in the text, and also an indicative $H-T$ diagram showing the points of the experimental measurements and simulations. (Bottom left) The experimental data from the left panel of Fig. 4 in Ref. [33]. these experimental data are composed of four scanning tunneling microscopy measurements at zero-bias conductance for the magnetic fields indicated inside these measurements. The vortices cores appear as small bright spots. (Bottom right) The simulation patterns with the respective vorticity next to each. The simulated patterns that can be compared with the experimental patterns are to the near right of the corresponding patterns. The simulation patterns are in the natural logarithmic scale that goes from red (dark gray) to yellow (light gray) to blue (dark gray). In the patterns is possible to see the center of the vortices as small blue (dark gray) spots in the center of the samples. (Top right) The $T-H$ diagram showing the superconductivity nucleation curve (in black), the experimental line (red), and the adjusted experimental line (see main text, dashed blue line). On top of these lines, small squares and open circles, with the same colors of the respective lines, indicate the points corresponding to the simulations and the experimental distributions. Dashed vertical lines indicate the magnetic flux of the simulated patterns. The vorticity and magnetic flux values of the simulated patterns were added on the top of the dashed lines. 
of each experimental measurement in the figure) for the vorticites $L=2,3,4$ are shown in the bottom-left panel of Fig. 10, and to their right we can find the simulated distributions for the adjusted values of $\Phi / \Phi_{0}$ (see below). We must keep in mind that the experimental data were taken at $T=T_{c} / 20$ and the simulation was made near the nucleation boundary, $T=T_{c}(H)$, as can be seen in the top-right plot of the same figure. For this sample, the nucleation point in the $T-H$ diagram corresponding to $T=T_{c} / 20$ is given by a magnetic flux of $\phi / \phi_{0} \approx 6.3$ (estimated from Fig. 3 of Ref. [33] and Fig. 1 of the Supplemental Materials for this publication), which corresponds to $A / \xi^{2} \approx 19.9$. The red line in the plot shows the temperature of the measurements (marked with red squares). The points of the measurements are not coherent with the nucleation curve, e.g., the experimental point $\Phi / \Phi_{0}=3.1$ has vorticity $L=2$ but the theoretical nucleation of $L=2$ is only at 3.4. An error in the estimation of the area can account for this discrepancy. An adjustment of the size of the sample by $20 \%$, as shown by the blue line in Fig. 10, can bring the experimental values back into the respective vorticity areas of the diagram.

We can see from Fig. 10 that the experimental point with $L=4$ is near nucleation, making it more comparable to simulation. We also note that the vortex distribution in the nucleation defines a lower bound for the vortex distances. Since the experimental data are not taken at the same temperature, we are only going to look to the vortex arrangement and not to the condensate density distribution. We note that the simulated condensate density distributions in Fig. 10 are in the log scale, in order to observe the vortex locations more easily.

We can see that the vortex patterns appear to be remarkably similar to the experimental ones for all values of the magnetic field. In simulations for squares and circles done previously [2], by lowering the temperature the vortex patterns maintain a similar vortex arrangement to $A / \xi^{2} \approx 20$. Lowering the temperature further, the symmetry of some of the patterns breaks. For some of the patterns, e.g., $L=4$ in a square, the vortex arrangement remains the same as at nucleation. This could be the explanation for the excellent agreement with the experimental data.

\section{CONCLUSIONS}

The proposed method extends naturally the previously developed superconducting gauge method. The use of basis functions that already satisfy the boundary conditions is a key element present in the original method and the extended one. These basis functions differ for each of these methods, but only few basis functions are needed to get a very good approximation of the solution in both methods. These methods were compared for the case of the triangle and the square geometries. The solutions of the extended method match the previous ones for these geometries.

With this new method, new geometries are now accessible to semianalytical treatment. The application of the latter in the present work allowed us to observe certain common features shared by the solutions of LGL equation calculated for regular polygons. We have applied also the method to a deformed hexagon and found that the solutions for this geometry differ markedly from the solutions for the regular hexagon. We have then extended the method to boundaries with smooth curves and applied this to an experimental sample. The results of the simulations were compared to the experimental distributions and have shown remarkable agreement.

The solutions of the LGL equation for different polygons presented here have demonstrated that the proposed method is efficient (uses only a few basis functions) and fairly accurate. Moreover, it can be equally applied to irregular polygons and smooth closed boundaries with comparable efficiency.

\section{ACKNOWLEDGMENTS}

The authors gratefully acknowledge financial support of the Methusalem program at the KU Leuven and the FWO grants of the Flemish Science Foundation.
[1] V. A. Schweigert, F. M. Peeters, and P. S. Deo, Phys. Rev. Lett. 81, 2783 (1998); V. A. Schweigert and F. M. Peeters, Phys. Rev. B 57, 13817 (1998).

[2] L. F. Chibotaru, A. Ceulemans, V. Bruyndoncx, and V. V. Moshchalkov, Nature (London) 408, 833 (2000).

[3] A. A. Abrikosov, Fundamental Theory of Metals (NorthHolland, Amsterdam, 1988).

[4] D. Saint-James, E. J. Thomas, and G. Sarma, Type II Superconductivity (Pergamon Press, Oxford, UK, 1969).

[5] V. V. Moshchalkov, X. G. Qiu, and V. Bruyndoncx, Phys. Rev. B 55, 11793 (1997).

[6] L. F. Chibotaru, G. Teniers, A. Ceulemans, and V. V. Moshchalkov, Phys. Rev. B 70, 094505 (2004).

[7] V. V. Moshchalkov, L. Gielen, C. Strunk, R. Jonckheere, X. Qiu, C. Van Haesendonck, and Y. Bruynseraede, Nature (London) 373, 319 (1995).

[8] L. F. Chibotaru, A. Ceulemans, V. Bruyndoncx, and V. V. Moshchalkov, Phys. Rev. Lett. 86, 1323 (2001).
[9] G. Teniers, L. F. Chibotaru, A. Ceulemans, and V. V. Moshchalkov, Europhys. Lett. 63, 296 (2003).

[10] R. Geurts, M. V. Milošević, and F. M. Peeters, Phys. Rev. B 81, 214514 (2010).

[11] J. Barba-Ortega, E. Sardella, and J. Albino Aguiar, J. Super. Nov. Mag. 24, 97 (2011).

[12] R. Geurts, M. V. Milošević, and F. M. Peeters, Phys. Rev. B 79, 174508 (2009).

[13] T. Mertelj and V. V. Kabanov, Phys. Rev. B 67, 134527 (2003).

[14] V. R. Misko, V. M. Fomin, J. T. Devreese, and V. V. Moshchalkov, Phys. Rev. Lett. 90, 147003 (2003).

[15] J. Bonča and V. V. Kabanov, Phys. Rev. B 65, 012509 (2001); (private communication).

[16] D. Saint-James, Phys. Lett. 15, 13 (1965).

[17] R. B. Dingle, Proc. R. Soc. London A 211, 500 (1952).

[18] L. F. Chibotaru, V. H. Dao, and A. Ceulemans, Europhys. Lett. 78, 47001 (2007).

[19] P. J. Pereira, L. F. Chibotaru, and V. V. Moshchalkov, Phys. Rev. B 84, 144504 (2011). 
[20] V. Bruyndoncx, L. Van Look, M. Verschuere, and V. V. Moshchalkov, Phys. Rev. B 60, 10468 (1999).

[21] L. F. Chibotaru, A. Ceulemans, G. Teniers, V. Bruyndoncx, and V. V. Moshchalkov, Eur. Phys. J. B 27, 341 (2002).

[22] L. F. Chibotaru, A. Ceulemans, M. Morlele, G. Teniers, C. Carballeira, and V. V. Moshchalkov, J. Math. Phys. 46, 095108 (2005).

[23] S. Kim, M. Gunzburger, J. Peterson, and C. Hu, Commun Comput. Phys. 6, 673 (2008).

[24] J. R. Kuttler and V. G. Sigillito, SIAM Rev. 26, 163 (1984).

[25] E. Lijnen, L. F. Chibotaru, and A. Ceulemans, Phys. Rev. E 77, 016702 (2008).

[26] L. Molinari, J. Phys. A 30, 6517 (1997).
[27] S. Panda, S. Chakraborty, and S. P. Khastgir, Eur. Phys. J. Plus 126, 62 (2011).

[28] P. Amore, J. Math. Phys. 51, 052101 (2010).

[29] T. A. Driscoll and L. N. Trefethen, Schwarz-Christoffel Mapping (Cambridge University Press, London, 2002).

[30] T. A. Driscoll, ACM T. Math. Software 22, 168 (1996).

[31] L. V. Kantorovich and V. I. Krylov, Approximate Methods of Higher Analysis (Interscience, New York, 1964).

[32] P. O. Löwdin, J. Chem. Phys. 18, 365 (1950).

[33] T. Cren, L. Serrier-Garcia, F. Debontridder, and D. Roditchev, Phys. Rev. Lett. 107, 097202 (2011). 